\title{
Youthful Brains in Older Adults: Preserved Neuroanatomy in the Default Mode and Salience Networks Contributes to Youthful Memory in Superaging
}

\author{
Felicia W. Sun, ${ }^{3,4 *}$ Michael R. Stepanovic, ${ }^{1,2,3 *}$ Joseph Andreano, ${ }^{3,4,5}$ Lisa Feldman Barrett, ${ }^{3,4,5}$ \\ Alexandra Touroutoglou, ${ }^{2,3 \dagger}$ and Bradford C. Dickerson ${ }^{1,2,3 \dagger}$ \\ ${ }^{1}$ Frontotemporal Disorders Unit, Massachusetts General Hospital and Harvard Medical School, Charlestown, Massachusetts 02129, ${ }^{2}$ Department of \\ Neurology, Massachusetts General Hospital and Harvard Medical School, Charlestown, Massachusetts 02114, ${ }^{3}$ Athinoula A. Martinos Center for Biomedical \\ Imaging, Massachusetts General Hospital and Harvard Medical School, Charlestown, Massachusetts 02114, and ${ }^{4}$ Psychiatric Neuroimaging Division, \\ Massachusetts General Hospital and Harvard Medical School, Charlestown, Massachusetts 02114, and ${ }^{5}$ Department of Psychology, Northeastern University, \\ Boston, Massachusetts 02115
}

Decline in cognitive skills, especially in memory, is often viewed as part of "normal" aging. Yet some individuals "age better" than others. Building on prior research showing that cortical thickness in one brain region, the anterior midcingulate cortex, is preserved in older adults with memory performance abilities equal to or better than those of people 20-30 years younger (i.e., "superagers"), we examined the structural integrity of two large-scale intrinsic brain networks in superaging: the default mode network, typically engaged during memory encoding and retrieval tasks, and the salience network, typically engaged during attention, motivation, and executive function tasks. We predicted that superagers would have preserved cortical thickness in critical nodes in these networks. We defined superagers (60 - 80 years old) based on their performance compared to young adults (18 - 32 years old) on the California Verbal Learning Test Long Delay Free Recall test. We found regions within the networks of interest where the cerebral cortex of superagers was thicker than that of typical older adults, and where superagers were anatomically indistinguishable from young adults; hippocampal volume was also preserved in superagers. Within the full group of older adults, thickness of a number of regions, including the anterior temporal cortex, rostral medial prefrontal cortex, and anterior midcingulate cortex, correlated with memory performance, as did the volume of the hippocampus. These results indicate older adults with youthful memory abilities have youthful brain regions in key paralimbic and limbic nodes of the default mode and salience networks that support attentional, executive, and mnemonic processes subserving memory function.

Key words: aging; cerebral cortex; default mode network; memory; salience network

Significance Statement

Memory performance typically declines with age, as does cortical structural integrity, yet some older adults maintain youthful memory. We tested the hypothesis that superagers (older individuals with youthful memory performance) would exhibit preserved neuroanatomy in key brain networks subserving memory. We found that superagers not only perform similarly to young adults on memory testing, they also do not show the typical patterns of brain atrophy in certain regions. These regions are contained largely within two major intrinsic brain networks: the default mode network, implicated in memory encoding, storage, and retrieval, and the salience network, associated with attention and executive processes involved in encoding and retrieval. Preserved neuroanatomical integrity in these networks is associated with better memory performance among older adults.

\section{Introduction}

As humans age, memory and many other cognitive functions often decline. When a neuropsychologist evaluates an older adult, "normal" performance is substantially lower than that of a younger adult. For example, on the California Verbal Learning Test (CVLT), an average 25-year-old remembers 14 words, while 
an average 75 year-old remembers 9 words, more than 2 SDs lower (Delis et al., 1987). Nevertheless, there is substantial variation in the degree of cognitive decline with age. Some older adults - referred to by one group as "superagers" - continue to perform at a level similar to middle-aged adults (Harrison et al., 2012; Rogalski et al., 2013; Gefen et al., 2014; Gefen et al., 2015), and sometimes even young adults (Weintraub et al., 1994). Investigation of the biological mechanisms associated with "youthful" cognitive function in such individuals is crucial to understanding "successful aging" (Depp and Jeste, 2006). In this study, we sought to replicate and extend prior work on superaging by testing hypotheses regarding the structural integrity of two key brain networks that contribute to memory function.

Memory requires that information be encoded, stored, and retrieved. To explicitly encode information, such as a list of words, an individual must first be motivated to attend to the relevant material, engage working memory, and organize the information (Wolk et al., 2011). Broadly speaking, these functions are subserved by fronto-parietal-cingulate circuitry, variously referred to as attentional (Corbetta and Shulman, 2002), executive (Dosenbach et al., 2006; Cole and Schneider, 2007), working memory (Koechlin et al. 1999; Gruber and Goschke 2004), and/or salience systems (Seeley et al. 2007; Touroutoglou et al., 2012). In conjunction with circuitry supporting semantic memory, this circuitry is engaged when new information is organized within the context of previously existing knowledge (Simons and Spiers, 2003; Squire, 2007). Once encoded, information is consolidated and stored as "long-term episodic memories," by way of the medial temporal lobe (MTL) memory system localized in the hippocampus, medial temporal cortex, and retrosplenial/posterior cingulate cortex (Squire et al., 2004), as well as other key nodes of the default mode network (Dickerson and Eichenbaum, 2010). When information is subsequently retrieved (e.g., during free recall of a word list), attentional, salience, executive, and semantic networks are engaged in conjunction with the MTL memory system; when any of these brain regions are lesioned, memory retrieval is impaired (Wolk et al., 2011).

Normal aging is well known to be accompanied by widespread reductions in the thickness of many of these brain regions (McGinnis et al., 2011; Bakkour et al., 2013), in parallel with age-related decline in memory function (McDaniel et al., 2008). Age-related atrophy is particularly prominent in key frontoparietal nodes of the working memory, executive, salience, and default mode circuitry, such as in lateral and medial prefrontal and lateral parietal cortices, as well as portions of the cingulate cortex and medial temporal lobe (McGinnis et al., 2011; Bakkour et al., 2013). Based on this summary of the processes that subserve memory function and our knowledge of age-related cortical changes, we hypothesized that superagers would exhibit "youthful" neuroanatomy

\footnotetext{
${ }^{\dagger}$ A.T., L.F.B, and B.C.D. contributed equally to this work. The authors declare no competing financial interests.

This article is freely available online through the J Neurosci Author Open Choice option.

Correspondence should be addressed to Dr. Alexandra Touroutoglou, Department of Neurology, Massachusetts General Hospital, 149 13th Street, Suite 2691, Charlestown, MA 02129. E-mail: atouroutoglou@mgh.harvard.edu; or Dr. Lisa Feldman Barrett, Department of Psychology, 125 Nightingale Hall, Northeastern University, Boston, MA 02115-5000. E-mail: I.barrett@northeastern.edu; or Dr. Bradford C. Dickerson, Frontotemporal Disorders Unit, Massachusetts General Hospital, 13th Street, Suite 2691, Charlestown, MA 02129. E-mail: brad.dickerson@mgh.harvard.edu.

D0I:10.1523/JNEUROSCI.1492-16.2016

Copyright $\odot 2016$ Sun, Stepanovic et al.

This is an Open Access article distributed under the terms of the Creative Commons Attribution License Creative Commons Attribution 4.0 International, which permits unrestricted use, distribution and reproduction in any medium provided that the original work is properly attributed.
}

within the networks summarized here. We further hypothesized that, within the entire group of cognitively normal older adults, the cortical thickness of these brain regions would predict individual differences in memory performance.

\section{Materials and Methods}

Participants. Ninety-one participants (48 males, 43 females) were recruited from the greater Boston area, comprising young adults (ages $18-35 ; 24$ males, 23 females) and elderly adults (ages 60-80;24 males, 20 females). They were phone screened to ensure MRI scan eligibility and compensated with payment for their completion of the study. All individuals were right-handed native English speakers and had normal or corrected-to-normal vision. No participant reported a history of neurological or psychiatric disorders. Additional exclusionary criteria included MRI safety incompatibility, substance dependence, and CNS-active medications.

Neuropsychological criteria for superaging. All participants were required to score within 1.5 SDs of published normative values for each neuropsychological instrument on the basis of their age and education. To be designated as a superager, an elderly adult was required to meet two strict psychometric criteria similar to previous studies (Harrison et al. 2012; Rogalski et al., 2013; Gefen et al., 2014). First, they were required to perform at or above the mean gender-adjusted value for young adults (age range, 18-32) on the Long Delay Free Recall measure of the CVLT (CVLT-LD). Second, they were required to perform no lower than $1 \mathrm{SD}$ below the mean for their age group on the Trail Making Test Part B (TMT-B) (Tombaugh, 2004). All older adults participants not meeting these criteria were designated as typical older adults.

Study procedures. Participants arrived at the lab on Day 1 to complete a neuropsychological battery of tests, including the CVLT (Delis et al., 1987); TMT (Reitan, 1958; Strauss et al., 2006); portions of the Executive Abilities: Measures and Instruments for Neurobehavioral Evaluation and Research (EXAMINER) battery (Kramer et al., 2014) including Flanker and the Continuous Performance Task, 1- and 2-back; and the Mini-Mental State Examination (MMSE; Folstein et al., 1975). On Day 2 (1-3 d later), participants completed a standardized MRI scan from which measurements of neuroanatomy were obtained.

MRI acquisition. MRI scans were collected on a 3 Tesla Tim Trio System using a 12 channel phased-array head coil (Siemens) and a standardized high resolution T1-weighted magnetization-prepared rapid gradient echo protocol optimized for gray-white contrast [repetition time (TR), $2530 \mathrm{~ms}$; echo time (TE), $3.48 \mathrm{~ms}$; flip angle (FA), $7^{\circ} ; 256 \times$ $256\left(1 \mathrm{~mm}^{2} \times 1 \mathrm{~mm}^{2}\right)$ in-plane resolution; 176 sagittal slices $(1 \mathrm{~mm}$ thickness)].

A priori functional network definition. As elaborated in the introduction, we used a hypothesis-driven approach focusing on two large-scale intrinsic connectivity brain networks known to be important for memory: the default mode network, as defined by AndrewsHanna et al. (2010), and the salience network, as defined by Touroutoglou et al. (2012) (networks illustrated in Fig. 1). We identified these networks by analyzing data from an independent sample of 89 young adults ( 44 men) ranging in age from 18 to 33 , with a mean age of 22.4 years (SD, 3.34) as detailed in the study by Touroutoglou et al. (2012). In brief, whole-brain resting-state fMRI data (TR, 3000 $\mathrm{ms}$; TE, $30 \mathrm{~ms}$; FA, $90^{\circ}$; $3.0 \mathrm{~mm}$ isotropic voxels; 47 slices; 2 runs of 124 time points) were preprocessed, and two spherical regions of interest (ROIs; $4 \mathrm{~mm}$ radius) were used as seed regions: the right dorsal anterior insula $(+36,21,1, \mathrm{MNI})$, to identify the salience network, and the posterior cingulate cortex $(-8,-56,26, \mathrm{MNI})$, to identify the default mode network. We computed Pearson's product moment correlations, $r$, between the mean signal time course of the seed ROIs and the time course of all voxels across the brain. The resulting correlation maps were converted to $z$ values, using Fisher's $r$-to- $z$ transformation, and were averaged across subjects. The two resultant correlation maps were projected to the FreeSurfer fsaverage surface and binarized at a threshold of $z(r)=0.2$ (Van Dijk et al., 2010). These two networks of interest were used as masks in the subsequent analysis comparing superagers to typical older adults. 


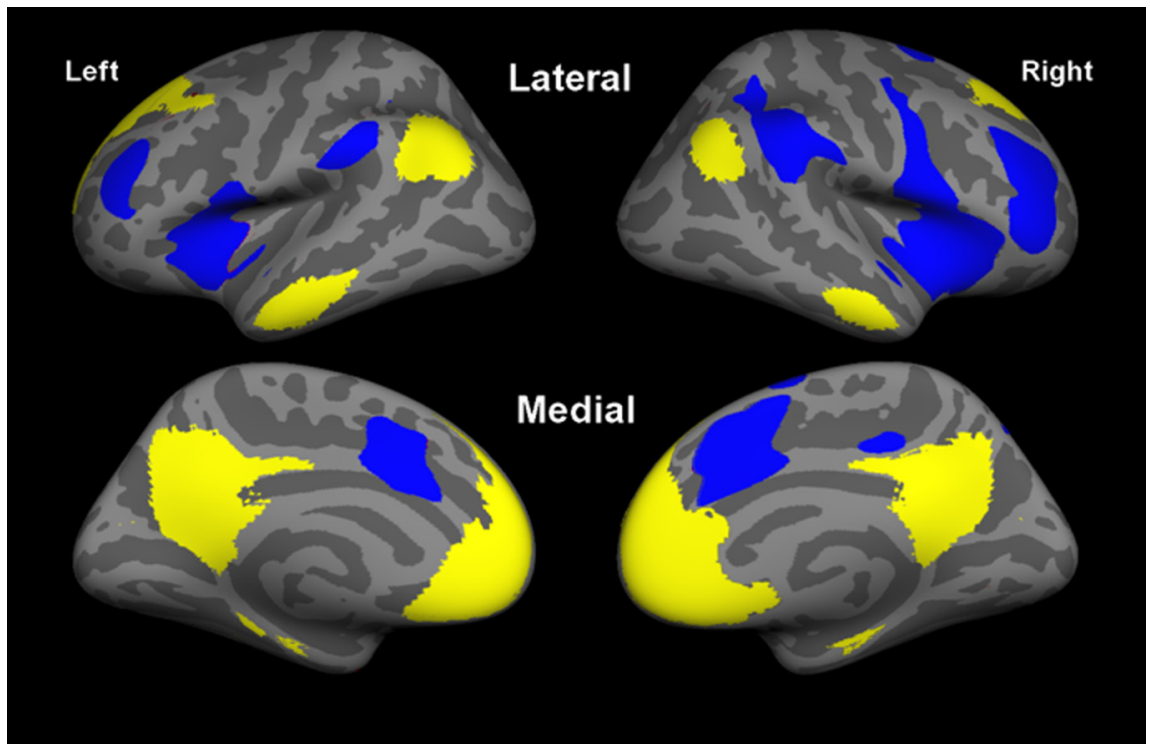

Figure 1. We used a network approach to test the hypothesis that preserved memory in superaging is associated with preserved structure in the salience network and default mode network. We used masks of the salience network (blue) anchored in the dorsa anterior insula (Touroutoglou et al., 2012) and the default mode network (yellow; Andrews-Hanna et al., 2010) in our primary analyses of preserved brain structure in superaging. For the binarized salience network, $z<0.2$, and for the default mode network $z<0.2$ (maps are shown on the "fsaverage" subject's inflated cortical surface). The general linear model analysis depicted in Figure 2 focuses only on cortex within these two networks.

Analysis of structural MRI data. FreeSurfer version 5.3 (http://surfer. nmr.mgh.harvard.edu) was used for all MRI data analysis. Each structural volume underwent spatial and intensity normalization, skull stripping, and an automated segmentation of cerebral white matter (Dale et al., 1999) to locate the gray-white boundary. Defects in the surface topology were corrected (Fischl et al., 2001), and the graywhite boundary was deformed outward using an algorithm designed to obtain an explicit representation of the pial surface. Cortical thickness was then derived from the distance between the gray-white boundary and the pial surface across the entire cortical mantle (Fischl and Dale, 2000). In accordance with a standardized, objective quality assurance protocol, the reconstructed cortical surface was visually inspected for technical accuracy and manually edited when necessary (minimal manual editing was required in the current study). This automated procedure is highly reliable across sessions and scanning platforms (Dickerson et al., 2008).

To identify regions of significantly thicker cortex in superagers than in typical older adults, we generated statistical surface maps using the general linear model analysis in FreeSurfer, comparing cortical thickness between the two groups for each vertex of the cortical surface within the network-of-interest masks. For this analysis, we used a statistical threshold of $p<0.05$ uncorrected. This mapdepicting locations on the cortical surface where superagers showed a thicker cortical ribbon than typical older adults (Fig. 2) —-was used to generate ROIs for subsequent brain-behavior analyses and to compare with younger adults; that is, for each cluster in Figure 2 that fell within the masked networks of interest, a ROI was made and the thickness at each vertex point within the ROI was averaged to determine the thickness of each individual subject's ROI.

To control for individual variability in thickness across the entire cortical mantle, mean thickness of the entire cortex was regressed out of the variance in mean thickness of each ROI. The resulting standardized residuals were used in correlation analyses to test the hypothesis that they relate to memory performance (CVLT-LD scores). To test the specificity of these relationships, four additional cortical ROIs were selected that did not belong to either large-scale functional network of interest, but rather were localized in primary sensory cortical regions not hypothesized to be related to memory function. Bonferroni corrections were used for these correlation analyses, resulting in a significance threshold of $p<0.005$.
Finally, we measured hippocampal volume adjusted for total intracranial volume (raw hippocampal volume derived from the FreeSurfer automated segmentation divided by estimated total intracranial volume) to test the hypothesis that the preservation of memory function in superaging was related to relatively preserved hippocampal volume. We also examined amygdala volume to investigate the specificity of these relationships.

\section{Results}

\section{Youthful behavioral performance in superaging}

Ten participants did not complete both the behavioral and scanning portions of the study, resulting in a final sample size of 81 individuals: 41 young adults (20 males, mean age, 25.1; SD, 3.62; $21 \mathrm{fe}$ males, mean age, $24.0 ; S D, 3.5)$ and 40 elderly adults (20 males, mean age, 66.5; $\mathrm{SD}, 6.27 ; 20$ females, mean age, 67.3; SD, 4.68). Of the 40 elderly participants, 17 were classified as superagers $(4$ males, mean age, 69.25 years; SD, $4.59 ; 13 \mathrm{fe}$ males, mean age, 67.38 years; SD, 1.4). The remaining 23 formed the "typical older adult" group (15 males, mean age, 65.00; SD, 5.88; 9 females, mean age, 67.71; SD, 5.08). All 41 young adults performed within the normative values for their age and gender on CVLT and TMT-B. Results are summarized in Table 1.

A one-way ANOVA confirmed that the three groups differed in episodic memory performance (CVLT-LD; $F_{(2,78)}=15.8$, $p<0.001)$ as well as executive functioning (TMT-B; $F_{(2,78)}=3.94$, $p<0.03)$. For CVLT-LD, post hoc comparisons using the Tukey HSD test indicated that superagers performed better than both young adults $(p<0.01)$ and typical older adults $(p<0.001)$. For TMT-B, superagers' performance did not differ from typical older adults $(p=0.53)$ or young adults $(p=0.40)$. However, typical older adults performed worse than young adults $(p<0.05)$.

Post hoc comparisons between the two older adult groups revealed that the superagers also performed better than typical older adults on CVLT Trial 1, Trial 5, total learning, Long Delay Free Recall intrusion rate, and Long Delay Free Recall semantic clustering, and showed a trend toward better performance on Long Delay Recognition memory accuracy. There were no differences between superagers and typical older adults on other psychometric measures. There were no differences in age $(p=0.50)$ or years of education $(p=0.30)$, but there was a trend toward a greater number of women in the superager group than in the typical older adult group $(p=0.08)$.

Post hoc comparisons between superagers and young adults revealed indistinguishable performance on TMT-A $(p=0.16)$, EXAMINER verbal fluency $(p=0.79)$ and category fluency $(p=$ $0.81)$, and the MMSE $(p=0.83)$ and the CVLT-LD semantic clustering ratio.

\section{Structural integrity of cortex within the default mode and salience networks}

Within the default mode and salience networks, superagers had statistically thicker cortex than typical older adults in a number of key regions (Fig. 2A). Regions with preserved 

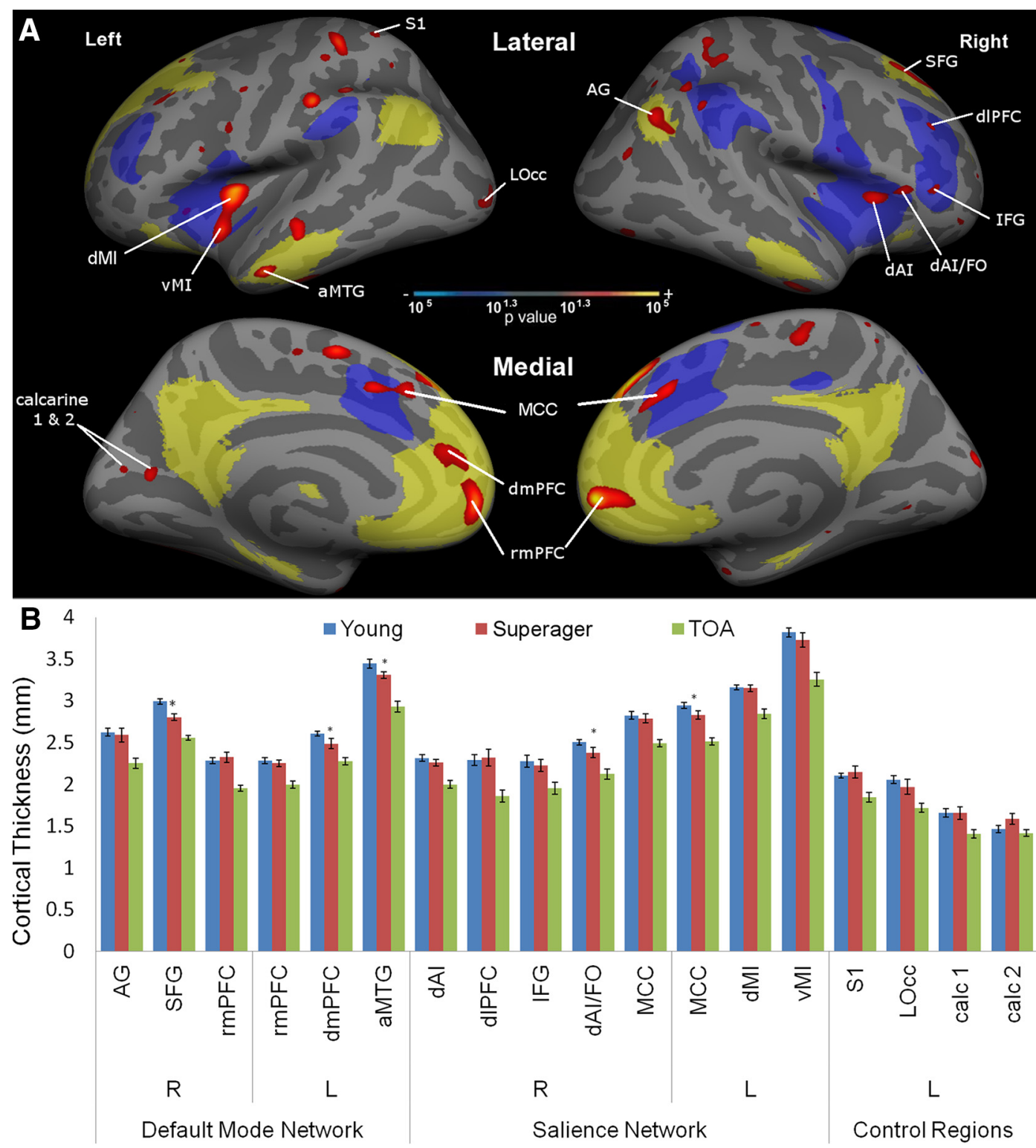

Figure 2. Regions of preserved cortical thickness in superagers within the default mode and salience networks. $A$, This statistical map shows regions where the cortex of superagers is thicker than in typical older adults ( $p<0.05$; depicted as a red-to-yellow heat gradient), highlighting regions within a priori hypothesized networks of interest (salience network in blue; default mode network in pale yellow). Four control regions in primary sensory cortical areas outside the networks of interest are labeled for comparison. $\boldsymbol{B}$, Bar graphs show mean cortical thickness within each region labeled in the map. Although by definition all of these regions are thicker in superagers than typical older adults (TOAs), some of them are thinner in superagers than in young adults (partial preservation indicated with asterisks), while all others are "youthful" in superagers (fully preserved cortical thickness relative to young adults). R, Right hemisphere; L, left hemisphere. Error bars indicate SE.

thickness within the default mode network included the right angular gyrus (AG), right superior frontal gyrus (SFG), left anterior middle temporal gyrus (aMTG), bilateral rostral medial prefrontal cortex (rmPFC), and left dorsomedial prefrontal cortex (dmPFC). Note that the left dmPFC region and the right $\mathrm{rmPFC}$ region both extend into the dorsal/pregenual cingulate sulcus. Regions with preserved thickness within the salience network included the bilateral midcingulate cortex (MCC) extending into paracingulate cortex, left midinsula (MI), right dorsal anterior insula (dAI), right frontal operculum (FO), right dorsolateral prefrontal cortex (dlPFC), and right inferior frontal gyrus (IFG). Preserved thickness in primary sensorimotor cortical zones, which we used here as control ROIs, included left primary somatosensory cortex (S1), left lateral occipital cortex (lOcc), and two calcarine cortical regions (calc 1 and calc 2 ).

Notably, some of these regions showed full preservation: they were not simply thicker in superagers than in typical older adults, but were actually statistically indistinguishable from young adults. In the default mode network, areas of full preservation include the right $A G$, right SFG, left dmPFC, left aMTG, and bilateral rmPFC. In the salience network, these included right $\mathrm{dAI}$, right $\mathrm{d} \mathrm{PFC}$, right $\mathrm{IFG}$, right $\mathrm{dAI} / \mathrm{FO}$, left dorsal midinsula (dMI), left ventral midinsula (vMI), and bilateral MCC. None of the control regions showed this degree of preservation - the thickness of all of them in superagers was intermediate between young adults and typical older adults, or what we call partial preservation. 
Table 1. Demographic information and neuropsychological data

\begin{tabular}{|c|c|c|c|c|c|c|}
\hline \multirow{2}{*}{$\frac{\text { Neuropsychological measure }}{N}$} & \multirow{2}{*}{$\begin{array}{l}\text { Young adult } \\
\text { (mean/SD) }\end{array}$} & \multirow{2}{*}{$\begin{array}{l}\begin{array}{l}\text { Superager } \\
\text { (mean/SD) }\end{array} \\
17\end{array}$} & \multirow{2}{*}{$\begin{array}{l}\text { Typical older } \\
\text { adult (mean/SD) } \\
23\end{array}$} & \multicolumn{3}{|c|}{ ANOVA post hoc comparisons } \\
\hline & & & & SA vs YA & TOA vs YA & SA vs TOA \\
\hline Sex (\% female) & $51.2 \%$ & $70.6 \%$ & $34.8 . \%$ & $S A>Y A^{* * *}$ & $\mathrm{TOA}<\mathrm{YA} \mathrm{A}^{* * *}$ & \\
\hline Age (years) & $24.5(3.6)$ & $67.8(6.0)$ & $66.2(5.1)$ & & & \\
\hline Education (years) & $16.0(2.2)$ & $17.2(2.2)$ & $16.2(2.0)$ & & & \\
\hline Trail Making Test A (s) & $23.0(9.4)$ & $28.8(7.4)$ & $28.3(9.6)$ & & & \\
\hline Trail Making Test B (s) & $51.2(17.0)$ & $59.0(12.8)$ & $66.3(30.3)$ & & $\mathrm{TOA}>\mathrm{YA}^{*}$ & \\
\hline CVLT Trial 1 (16) & $8.6(2.2)$ & $9.7(1.7)$ & $7.3(2.5)$ & & & $\mathrm{SA}>\mathrm{TOA} * *$ \\
\hline CVLT Trial 5 (16) & $14.0(1.8)$ & $14.7(1.2)$ & $12.0(2.6)$ & & $\mathrm{TOA}<\mathrm{YA} \mathrm{A}^{* * *}$ & $\mathrm{SA}>\mathrm{TOA}^{* * *}$ \\
\hline CVLT total learning (80) & $60.9(9.6)$ & $65.9(4.8)$ & $51.7(11.1)$ & & $\mathrm{TOA}<\mathrm{YA} \mathrm{A}^{* *}$ & $\mathrm{SA}>\mathrm{TO} \mathrm{A}^{* * *}$ \\
\hline CVLT List B (16) & $8.3(2.1)$ & $7.4(2.3)$ & $6.55(2.5)$ & & $\mathrm{TOA}<\mathrm{YA} * *$ & \\
\hline CVLT List B intrusions & $0.20(0.46)$ & $0.18(0.53)$ & $0.45(0.60)$ & & & \\
\hline CVLT Long Delay Free Recall (16) & $13.2(2.2)$ & $15.0(0.9)$ & $11.0(2.2)$ & $S A>Y A^{* *}$ & TOA $<Y A^{* *}$ & $S A>T O A^{* * *}$ \\
\hline CVLT Long Delay Free Recall intrusions & $0.28(0.55)$ & $0.29(0.47)$ & $0.86(0.91)$ & & $\mathrm{TOA}>\mathrm{YA}^{* *}$ & $\mathrm{SA}<\mathrm{TOA}{ }^{*}$ \\
\hline CVLT recognition rate & $97.0 \%$ & $98.3 \%$ & $94.1 \%$ & & & $\mathrm{SA}>\mathrm{TOA}^{\dagger}$ \\
\hline CVLT Long Delay Free Recall semantic cluster ratio (4) & $3.1(0.9)$ & $3.7(0.6)$ & $2.8(1.3)$ & & & $\mathrm{SA}>\mathrm{TOA} *$ \\
\hline Flanker congruent (s) & $0.7(0.2)$ & $1.1(0.5)$ & $1.0(0.3)$ & $S A>Y A^{* * *}$ & $\mathrm{TOA}>\mathrm{YA}^{* * *}$ & \\
\hline Flanker incongruent (s) & $0.8(0.2)$ & $1.2(0.6)$ & $1.2(0.3)$ & $S A>Y A^{* * *}$ & $T O A>Y A^{* * *}$ & \\
\hline Flanker congruent error rate & $0.0 \%$ & $0.8 \%$ & $0.8 \%$ & & & \\
\hline Flanker incongruent error rate & $0.0 \%$ & $0.8 \%$ & $1.2 \%$ & & & \\
\hline Continuous performance task (s) & $0.53(0.09)$ & $0.65(0.07)$ & $0.66(0.10)$ & $S A>Y A^{* * *}$ & $T O A>Y A^{* * *}$ & \\
\hline 1-back $\left(d^{\prime}\right)$ & $2.6(0.7)$ & $2.5(0.8)$ & $2.4(0.6)$ & & & \\
\hline 2-back $\left(d^{\prime}\right)$ & $1.5(1.3)$ & $1.5(1.0)$ & $1.3(0.9)$ & & & \\
\hline Verbal fluency score & $31.2(8.2)$ & $32.8(7.1)$ & $31.2(9.5)$ & & & \\
\hline Category fluency score & $37.3(8.8)$ & $38.7(6.3)$ & $36.9(7.2)$ & & & \\
\hline Mini-Mental State Examination (30) & $29.2(0.8)$ & $29.7(0.5)$ & $28.8(1.2)$ & & & \\
\hline
\end{tabular}

CVLT total learning is the sum of items learned across all encoding trials. Intrusions are words freely recalled that were not on the appropriate list. Recognition rate is the percent correctly recognized. Semantic cluster ratio indicates the degree to which items within each of the four semantic categories were recalled together. Values in parentheses are the maximum score unless indicated otherwise. $s$, seconds; $d^{\prime}$, sensitivity index computed as $Z$ (proportion of false alarms)$Z$ (proportion of hits); $S A$, Superager; TOA, typical older adult; $Y$ A, young adult.

${ }^{*} p<0.05 ;{ }^{* *} p<0.01 ;{ }^{* * *} p<0.001 ;{ }^{\dagger} p<0.10$

As expected, there were volumetric differences between the three groups in the left hippocampus $\left(F_{(2,35.23)}=11.22, p<\right.$ $0.001)$, right hippocampus $\left(F_{(2,29.96)}=9.75, p<0.01\right)$, left amygdala $\left(F_{(2,33.94)}=9.58, p<0.01\right)$, and right amygdala $\left(F_{(2,33.47)}=8.33, p<0.01\right)$. Tukey post hoc tests indicated that typical older adults had smaller left and right hippocampal volume $(p<0.001)$ compared to young adults, while superagers showed full preservation (left, $p=0.15$; right, $p=0.96$ ). Superagers also had larger hippocampal volume than typical older adults in the right hemisphere $(p<0.01)$ but not the left $(p=$ 0.14 ). For the amygdala, typical older adults showed smaller amygdala volume compared to young adults (left, $p<0.01$; right, $p<0.01)$. Amygdala volumes in superagers showed small or trend-level differences from those of young adults (left, $p<0.05$; right, $p=0.07)$, but were similar to those of typical older adults (left, $p=0.71$; right, $p=0.59$ ).

\section{Relationship between morphometry and memory performance in preserved brain structures}

Within the entire group of older adults, the cortical thickness of some of the ROIs reported above predicted CVLT-LD performance (Table 2). In the default mode network, the regions where cortical thickness correlated with memory performance included the right SFG $(r=0.60)$, right and left $\operatorname{rmPFC}(r=$ 0.45 and $r=0.41$; Fig. 3$)$, and left aMTG $(r=0.41)$. Hippocampal volume also correlated with CVLT-LD $(r=0.40$; Fig. 4). In contrast, amygdala volume showed no relationship to memory $(r=0.17, p=0.3)$. In the salience network, regions where thickness correlated with memory performance included right and left MCC ( $r=0.55$ and $r=0.38)$, left ventral and dorsal midinsula $(r=0.45$ and $r=0.39)$, right dorsolateral PFC $(r=0.34)$, and right dorsal anterior insula $(r=0.34)$.
Table 2. Relationship between memory and cortical thickness in anatomical regions within networks of interest

\begin{tabular}{ll}
\hline Regions of interest & $\begin{array}{l}\text { Memory performance (CVLT-LD), Pearson } \\
\text { correlation coefficient }(r)\end{array}$ \\
\hline $\begin{array}{l}\text { Default mode network } \\
\text { R AG }\end{array}$ & 0.20 \\
R SFG & $0.60^{* * *}$ \\
R rmPFC & $0.45^{* * *}$ \\
L rmPFC & $0.41^{* *}$ \\
L dmPFC & 0.29 \\
L aMTG & $0.41^{* *}$ \\
Salience network & \\
R dAl & $0.34^{*}$ \\
R dIPFC & $0.34^{*}$ \\
R IFG & 0.24 \\
R dAI/F0 & 0.32 \\
R MCC & $0.54^{* * *}$ \\
L MCC & $0.38^{*}$ \\
L dMI & $0.39^{*}$ \\
L vMl & $0.45^{* * *}$ \\
Control regions & \\
L S1 & 0.27 \\
L LOcC & 0.10 \\
L calc 1 & 0.30 \\
L calc 2 & 0.14
\end{tabular}

Regions that survived Bonferroni corrections $(p<0.005)$ include the right SFG, right rmPFC, right MCC, and left vMI. Regions of interest listed here also appear in Figure 3. R, Right hemisphere; L, left hemisphere. ${ }^{*} p<0.05 ;{ }^{* *} p<0.01 ;{ }^{* * *} p<0.001$

Regions selected as control ROIs did not show relationships to memory (Table 2; Fig. 3). There was no relationship between memory performance and morphometry of the above regions of interest in the young adults. 

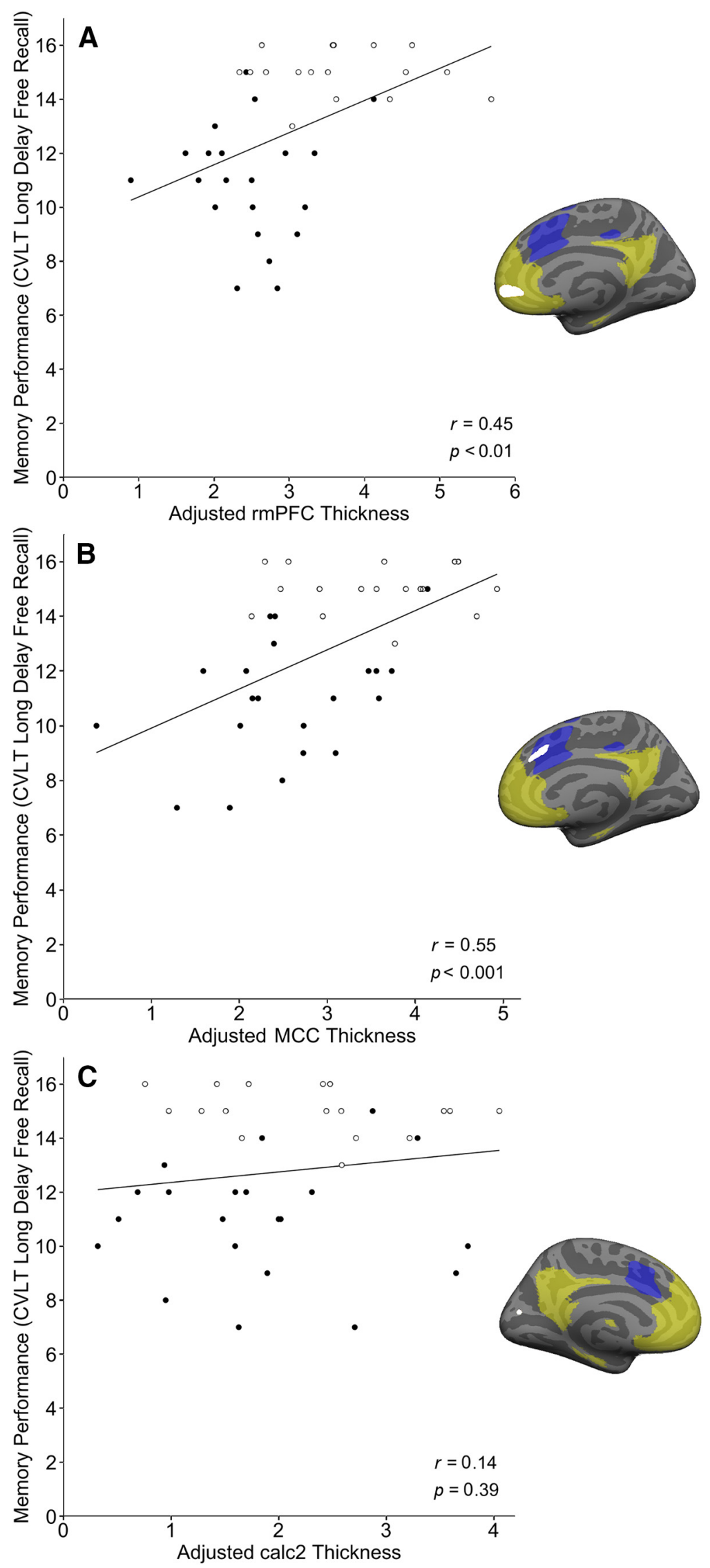

Figure 3. Preserved cortical thickness in some regions within default mode and salience networks supports preserved memory in elderly adults. The brain maps adjacent to scatter plots show regions where the cortex of superagers is thicker than in typical older adults ( $p<0.05$; depicted as solid white), highlighting the hypothesized networks of interest (salience network in blue;

\section{Discussion}

In this study, we extended prior work on superaging by demonstrating that the youthful memory abilities of superagers are associated with preserved neuroanatomical integrity in two brain networks that contribute to memory function. Based in part on our prior work, we focused on the default mode network (Miller et al., 2008; Wang et al., 2010), well known to be involved in episodic memory function, and the salience network (Touroutoglou et al., 2012), implicated in attention, executive control, and motivational and inhibitory processes integral to memory encoding and retrieval. Several regions in these networks showed partially preserved neuroanatomy in superagers compared to typical older adults; in fact, in some of these regions, superagers showed full preservation, where their thickness was statistically indistinguishable from young adults.

\section{Expanding the definition of superagers and probing their behavioral characteristics}

Harrison et al. (2012), Rogalski et al. (2013), and Gefen et al. (2014, 2015) coined the term superagers to refer to people over age 80 who perform at or above normative values for individuals in their 50s and 60s on a verbal long delay free recall test. We expanded the definition to a younger group of older adults, near or just older than typical retirement age $(60-80$ years old), in part because prior research has shown an inflection point in some cognitive abilities including memory at around age 65 (McDaniel et al., 2008; Rogalski et al., 2013). Rather than comparing them to middle-aged adults, we sought to identify older adults with youthful memory abilities at or above normative performance for 18- to 32-year-olds on the CVLT Long Delay Free Recall test.

We interpret the psychometric data shown in Table 1 as showing that superagers perform better than typical older

default mode network in pale yellow). Scatterplots in $\boldsymbol{A}$ and $\boldsymbol{B}$ illustrate the correlation between memory performance in the entire older adult group (superagers indicated by hollow points) and adjusted cortical thickness (standardized residuals after regressing out global mean cortical thickness, plus a constant) in the rostral medial prefrontal $(\boldsymbol{A} ; r=0.45, p<0.01)$ and the midcingulate cortex $(\boldsymbol{B} ; r=0.55, p<0.001)$. The scatterplot in $C$ shows no such relationship between memory performance and cortical thickness within one of the sensory regions used as a control. 
A

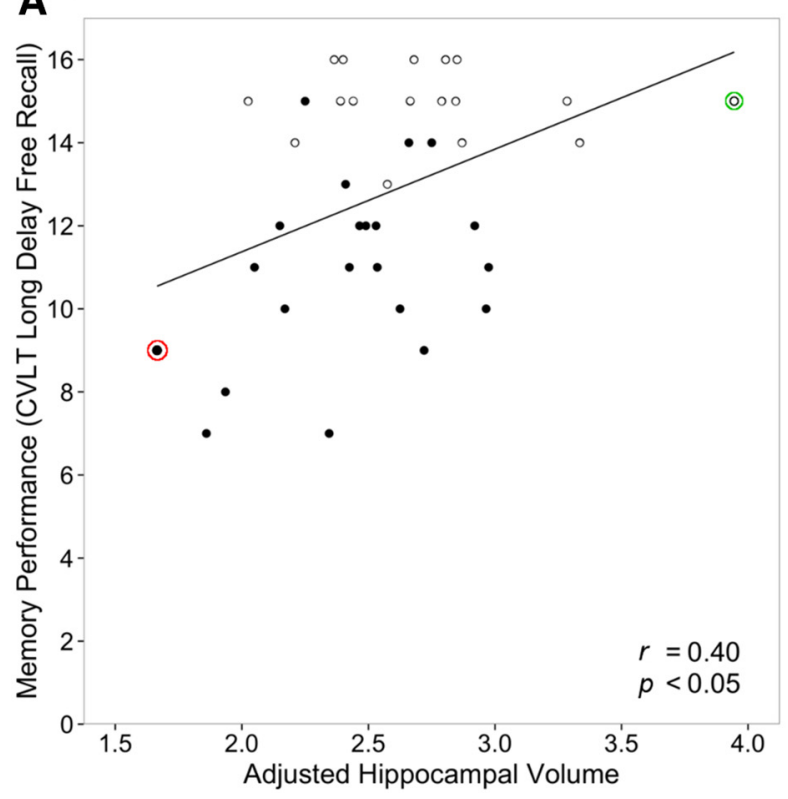

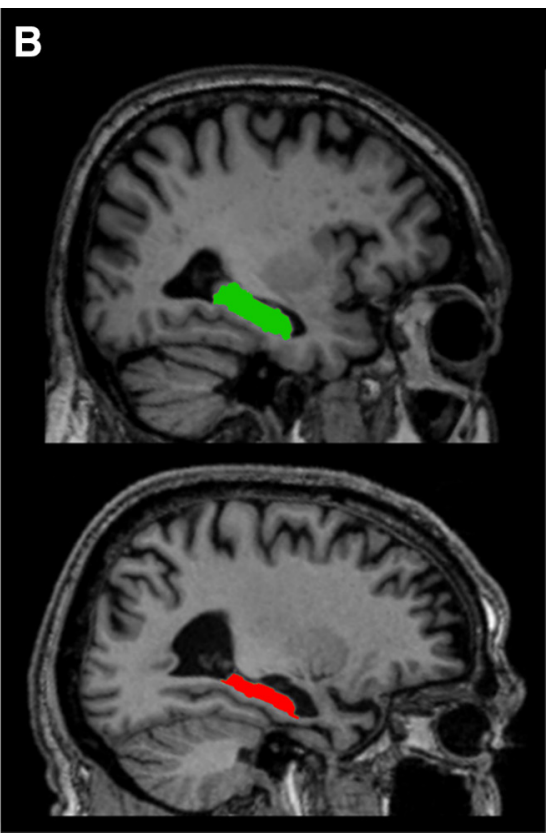

Figure 4. Preserved hippocampal volume—a key node in the default mode network— correlates with preserved memory in elderly adults. A, Larger total hippocampal volume (sum of left and right hemispheres divided by total intracranial volume; as depicted, multiplied by a constant) is correlated with better episodic memory performance in the entire group of older adults (superagers indicated by hollow points). $\boldsymbol{B}$, Parasagittal T1-weighted MRI scans at the level of the long axis of the hippocampus illustrate the effect shown in the scatterplot by showing the individuals with the largest (green) and smallest (red) hippocampal volume within the entire older adult sample. The individual with the largest hippocampal volume (green) is an 81-year-old male, has 20 years of education, and was able to freely recall 15 of the 16 words on the CVLT after the long delay. The individual with the smallest hippocampal volume (red) is a 74 -year-old male, has 16 years of education, and was able to freely recall 9 of the 16 words on the CVLT after the long delay.

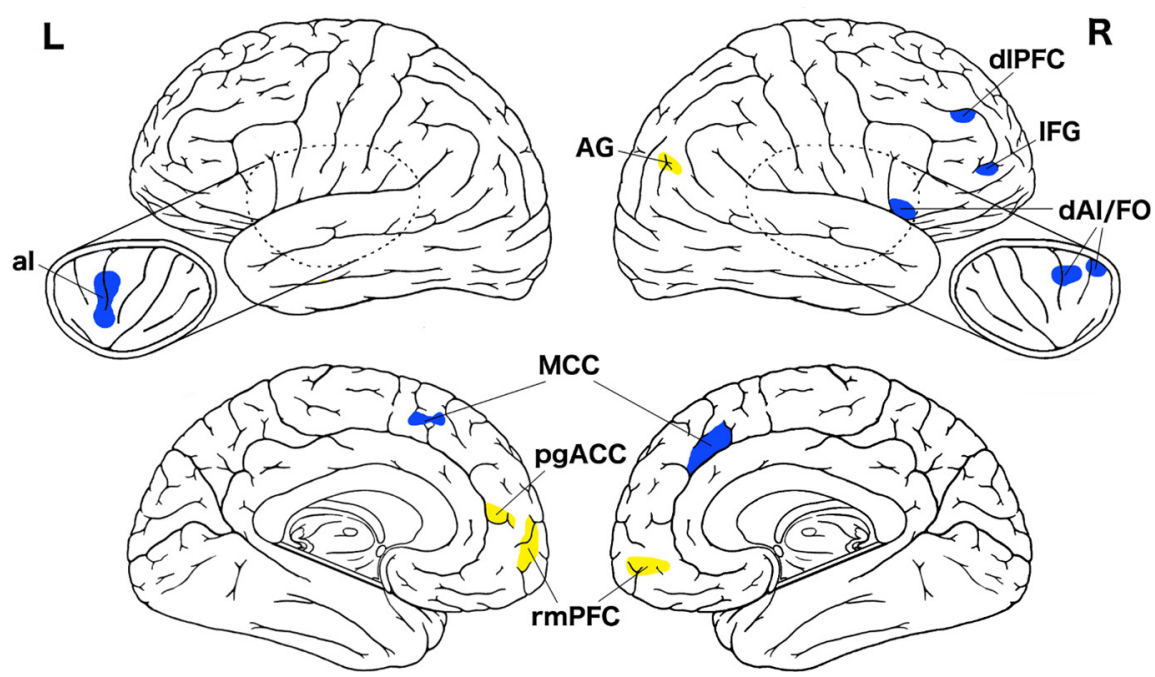

Figure 5. Superaging signature. The figure shows key nodes of the salience network (blue) and default mode network (yellow) where superagers and young adults are indistinguishable in cortical thickness. Preserved thickness in these regions is what distinguishes superagers from typical older adults.

adults in complex attentional and executive control processes involved in memory encoding and retrieval, in addition to having slightly superior performance in mnemonic storage processes. On the CVLT, superagers encode more items on Trial 1 and across all five trials than typical older adults, indicating superior auditory-verbal working memory, complex attention, and controlled memory encoding during the learning phase. In addition, a higher semantic clustering score indicates that executive control strategies taking advantage of the semantic relationships among these words are used to a greater degree in superagers than in typical older adults. In fact, superagers demonstrate slightly higher scores than young adults, suggesting that they may rely on such controlled encoding processes to a greater degree than young adults. While typical older adults make more intrusion errors than young adults, superagers do not, indicating better source memory and inhibitory control processes. The trend toward superior recognition discriminability scores in superagers relative to typical older adults may be an indication that mnemonic storage processes are more efficient, but this recognition memory test is likely not sensitive enough to detect differences in temporolimbic memory storage processes. These behavioral characteristics of superagers support our predictions that their superior memory likely relates to both memory storage processes subserved by temporolimbic episodic memory circuitry and attentional/executive encoding and retrieval processes subserved in part by the salience network. We have made complementary observations regarding the neuroanatomy of deficits in memory processes in patients with $\mathrm{AD}$ (Wolk et al., 2011), but to our knowledge these process-network hypotheses have not been investigated in older adults with superior memory.

\section{Neural network integrity as a neurobiological substrate for superaging}

We found support for our hypothesis regarding the structural integrity of the default mode and salience networks, with superagers showing much less atrophy than typical older adults in key 
nodes of these networks, which we refer to as the "superaging signature" (Fig. 5). Remarkably, this group of superagers exhibited full preservation of cortical thickness in several of these regions. Prior studies on the cortical neuroanatomy of superagers has called attention to the preservation of thickness of part of the cingulate cortex - a nearly identical anterior midcingulate region as we observed here (Harrison et al., 2012; Rogalski et al., 2013; Gefen et al., 2015). The comparison of superagers to typical older adults shown in Figure 1d of Harrison et al. (2012) demonstrates preserved thickness in some of the other regions we found, including rostral medial PFC, anterior insula, superior frontal gyrus, and angular gyrus.

\section{Relationship of cortical thickness within superaging signature to preserved memory}

Within the superaging signature, eight regions-the superior frontal gyrus, MCC, rmPFC, ventral midinsula, anterior middle temporal gyrus, dorsolateral PFC, dorsal anterior insula, and hippocampus-predict CVLT delayed free recall memory performance in the entire group of older adults. Prior work on superaging has reported relationships between global cortical volume and memory (Harrison et al., 2012) and between anterior midcingulate cortical thickness and memory (Gefen et al., 2015).

Our findings reiterate the importance for memory function of the integrity of regions typically considered to fall within the default mode network, including structures well known to be critical for memory such as the hippocampus. While the integrity of the hippocampus in aging is often viewed as an important factor determining memory abilities, its contribution to "youthful" memory function in older adults has not received previous attention. Prior studies on superaging have not reported on the hippocampus. Here, we found that superagers had fully preserved hippocampal volume, while typical older adults exhibited smaller volumes, as is commonly seen in older individuals. We also examined entorhinal, perirhinal, and parahippocampal cortical thickness and found that these were not preserved in superaging, nor were they correlated with memory in the entire older adult sample (data not shown). Other areas within the default mode network that subserve youthful memory performance include the rostral medial PFC, SFG, and anterior MTG. The mPFC has been observed as activated when people make responses that indicate a "feeling of knowing," or metacognitive experience of awareness of memory (Schnyer et al., 2004; Elman et al., 2012). The SFG contributes to generative, organizational processing during encoding (Fletcher et al., 1998; Wagner, 1999) as well as retrieval-related cue specification and generation of search strategies, along with manipulation and monitoring of search results. The anterior MTG is involved in semantic processing that enables deep encoding and interacts with ventrolateral PFC to enable semantic strategies for organizing material during encoding and retrieval (Martin and Chao, 2001; Simons and Spiers, 2003), and activity in the anterior temporal cortex has also been observed during feeling of knowing judgments (Elman et al., 2012).

In the salience network, cortical thickness in dAI, midinsula, midcingulate cortex, and dIPFC correlated with memory performance. Based on functional neuroimaging studies, dIPFC is involved in both encoding and retrieval, while other salience network regions, particularly the insula and anterior midcingulate cortex, are engaged prominently during retrieval (Wheeler et al., 1997; Konishi et al., 2000; Wheeler and Buckner, 2003), particularly effortful forms of retrieval such as free recall. For exam- ple, in an fMRI study of older adults, Dhanjal and Wise (2014) demonstrate robust activation during free recall in the inferior frontal gyrus, anterior temporal cortex, anterior insula, and anterior midcingulate gyrus in a region overlapping with that presently reported (Dhanjal and Wise, 2014; Figs. 2A,4C). Literature on cognitive control calls attention to the ubiquitous activation of these regions (Nelson et al., 2010) during the maintenance of set and monitoring of performance (Niendam et al., 2012; Power and Petersen, 2013), with slightly more dorsal frontoparietal regions being more engaged by task set selection and initiation (Power and Petersen, 2013). The anterior midcingulate cortex lies at the intersection of the dorsal and ventral salience subsystems (Touroutoglou et al., 2012) and is not only involved in cognitive control, but also correlated with affective experience (Touroutoglou et al., 2012; Lindquist et al., 2016; Raz et al., 2016) and, in particular, the affective/autonomic components of motivated task performance (Parvizi et al., 2013). This part of the cingulate cortex has also been conceptualized as estimating the "cost" associated with allocating control to a given task (Shenhav et al., 2013; Inzlicht et al., 2015). Thus, older adults with preserved anterior midcingulate cortical neuroanatomy may be particularly well positioned to generate and manage an affective response appropriate to an imminent challenge and allocate $\operatorname{cog}$ nitive control resources accordingly, which may support youthful memory function.

\section{Future directions}

One question underlying superaging is whether elite performance reflects a high baseline as opposed to or in conjunction with resilience against age-related decline; barring longitudinal assessment, it is impossible to know if these elderly adults were also top performers in their youth (Weintraub et al., 1994). Future epidemiological studies are also needed to estimate the prevalence and demographic characteristics in the general population. Although we have now replicated the observation that preserved structure of the MCC is important in superaging, future studies should attempt to replicate our other observations (e.g., hippocampal volume) in independent samples. Although high educational attainment has been shown to be a predictor of successful cognitive aging, education level does not differ between our superagers and typical older adults in this or previous studies. Future research should focus on identifying factors that play a role in preserved cognition, a number of which are already under investigation, such as exercise, diet, social activities, and genetic factors (Barnes et al., 2007; Habib et al., 2007; Yaffe et al., 2009; Josefsson et al., 2012).

\section{Notes}

Supplemental material for this article is available at http://dickersonlab.nmr. mgh.harvard.edu/Sun_SuperAging_Supplementary_Materials.pdf. The figure shows that superagers exhibit less cortical atrophy than typical older adults compared to young adults. This material has not been peer reviewed.

\section{References}

Andrews-Hanna JR, Reidler JS, Sepulcre J, Poulin R, Buckner RL (2010) Functional-anatomic fractionation of the brain's default network. Neuron 65:550-562.

Bakkour A, Morris JC, Wolk DA, Dickerson BC (2013) The effects of aging and Alzheimer's disease on cerebral cortical anatomy: specificity and differential relationships with cognition. Neuroimage 76:332-344. CrossRef Medline

Barnes DE, Cauley JA, Lui LY, Fink HA, McCulloch C, Stone KL, Yaffe K (2007) Women who maintain optimal cognitive function into old age. J Am Geriatr Soc 55:259-264. CrossRef Medline 
Cole MW, Schneider W (2007) The cognitive control network: Integrated cortical regions with dissociable functions. Neuroimage 37:343-360. CrossRef Medline

Corbetta M, Shulman GL (2002) Control of goal-directed and stimulusdriven attention in the brain. Nat Rev Neurosci 3:201-215. Medline

Dale AM, Fischl B, Sereno MI (1999) Cortical surface-based analysis. Neuroimage 9:179-194. CrossRef Medline

Delis DC, Kramer JH, Kaplan E. and Ober BA (1987) California Verbal Learning Test, Research Ed., manual. San Antonio, Texas: Harcourt Brace Jovanovich.

Depp CA, Jeste DV (2006) Definitions and predictors of successful aging: a comprehensive review of larger quantitative studies. Am J Geriatr Psychiatry 14:6-20. CrossRef Medline

Dhanjal NS, Wise RJ (2014) Frontoparietal cognitive control of verbal memory recall in Alzheimer's disease. Ann Neurol 76:241-251. CrossRef Medline

Dickerson BC, Eichenbaum H (2010) The episodic memory system: neurocircuitry and disorders. Neuropsychopharmacology 35:86-104. CrossRef Medline

Dickerson BC, Fenstermacher E, Salat DH, Wolk DA, Maguire RP, Desikan R, Pacheco J, Quinn BT, Van der Kouwe A, Greve DN, Blacker D, Albert MS, Killiany RJ, Fischl B (2008) Detection of cortical thickness correlates of cognitive performance: reliability across MRI scan sessions, scanners, and field strengths. Neuroimage 39:10-18.

Dosenbach NU, Visscher KM, Palmer ED, Miezin FM, Wenger KK, Kang HC, Burgund ED, Grimes AL, Schlaggar BL, Petersen SE (2006) A core system for the implementation of task sets. Neuron 50:799-812. CrossRef Medline

Elman JA, Klostermann EC, Marian DE, Verstaen A, Shimamura AP (2012) Neural correlates of metacognitive monitoring during episodic and semantic retrieval. Cogn Affect Behav Neurosci 12:599-609. CrossRef Medline

Fischl B, Dale AM (2000) Measuring the thickness of the human cerebral cortex from magnetic resonance images. Proc Natl Acad Sci U S A 97:11050-11055. CrossRef Medline

Fischl B, Liu A, Dale AM (2001) Automated manifold surgery: constructing geometrically accurate and topologically correct models of the human cerebral cortex. IEEE Trans Med Imaging 20:70-80. CrossRef Medline

Fletcher PC, Shallice T, Dolan RJ (1998) The functional roles of prefrontal cortex in episodic memory. I. Encoding. Brain 121:1239-1248. CrossRef Medline

Folstein MF, Folstein SE, McHugh PR (1975) “Mini-mental state.” A practical method for grading the cognitive state of patients for the clinician. J Psychiatr Res 12:189-198. CrossRef Medline

Gefen T, Shaw E, Whitney K, Martersteck A, Stratton J, Rademaker A, Weintraub S, Mesulam MM, Rogalski E (2014) Longitudinal neuropsychological performance of cognitive SuperAgers. J Am Geriatr Soc 62: 1598-1600. CrossRef Medline

Gefen T, Peterson M, Papastefan ST, Martersteck A, Whitney K, Rademaker A, Bigio EH, Weintraub S, Rogalski E, Mesulam MM, Geula C (2015) Morphometric and histologic substrates of cingulate integrity in elders with exceptional memory capacity. J Neurosci 35:1781-1791. CrossRef Medline

Gruber O, Goschke T (2004) Executive control emerging from dynamic interactions between brain systems mediating language, working memory and attentional processes. Acta psychologica 115:105-121.

Habib R, Nyberg L, Nilsson LG (2007) Cognitive and non-cognitive factors contributing to the longitudinal identification of successful older adults in the betula study. Neuropsychol Dev Cogn B Aging Neuropsychol Cogn 14:257-273. CrossRef Medline

Harrison TM, Weintraub S, Mesulam MM, Rogalski E (2012) Superior memory and higher cortical volumes in unusually successful cognitive aging. J Int Neuropsychol Soc 18:1081-1085. CrossRef Medline

Inzlicht M, Bartholow BD, Hirsh JB (2015) Emotional foundations of cognitive control. Trends Cogn Sci 19:126-132. CrossRef Medline

Josefsson M, de Luna X, Pudas S, Nilsson LG, Nyberg L (2012) Genetic and lifestyle predictors of 15 -year longitudinal change in episodic memory. J Am Geriatr Soc 60:2308-2312. CrossRef Medline

Koechlin E, Basso G, Pietrini P, Panzer S, Grafman J (1999) The role of the anterior prefrontal cortex in human cognition. Nature 399:148-151.

Konishi S, Wheeler ME, Donaldson DI, Buckner RL (2000) Neural cor- relates of episodic retrieval success. Neuroimage 12:276-286. CrossRef Medline

Kramer AD, Guillory JE, Hancock JT (2014) Experimental evidence of massive-scale emotional contagion through social networks. Proc Natl Acad Sci U S A 111:8788-8790. CrossRef Medline

Lindquist KA, Satpute AB, Wager TD, Weber J, Barrett LF (2016) The brain basis of positive and negative affect: evidence from a meta-analysis of the human neuroimaging literature. Cereb Cortex 26:1910-1922. CrossRef Medline

Martin A, Chao LL (2001) Semantic memory and the brain: structure and processes. Curr Opin Neurobiol 11:194-201. CrossRef Medline

McDaniel MA, Einstein GO, Jacoby LL (2008) New considerations in aging and memory: the glass may be half full. In: The handbook of aging and cognition (Craik FI, Salthouse T, eds), pp 255-310. New York: Psychology.

McGinnis SM, Brickhouse M, Pascual B, Dickerson BC (2011) Age-related changes in the thickness of cortical zones in humans. Brain Topogr 24: 279-291. CrossRef Medline

Miller SL, Celone K, DePeau K, Diamond E, Dickerson BC, Rentz D, Pihlajamäki M, Sperling RA (2008) Age-related memory impairment associated with loss of parietal deactivation but preserved hippocampal activation. Proc Natl Acad Sci U S A 105:2181-2186. CrossRef Medline

Nelson SM, Dosenbach NU, Cohen AL, Wheeler ME, Schlaggar BL, Petersen SE (2010) Role of the anterior insula in task-level control and focal attention. Brain Struct Funct 214:669-680. CrossRef Medline

Niendam TA, Laird AR, Ray KL, Dean YM, Glahn DC, Carter CS (2012) Meta-analytic evidence for a superordinate cognitive control network subserving diverse executive functions. Cogn Affect Behav Neurosci 12: 241-268. CrossRef Medline

Parvizi J, Rangarajan V, Shirer WR, Desai N, Greicius MD (2013) The will to persevere induced by electrical stimulation of the human cingulate gyrus. Neuron 80:1359-1367. CrossRef Medline

Power JD, Petersen SE (2013) Control-related systems in the human brain. Curr Opin Neurobiol 23:223-228. CrossRef Medline

Raz G, Touroutoglou A, Wilson-Mendenhall C, Gilam G, Lin T, Gonen T, Jacob Y, Atzil S, Admon R, Bleich-Cohen M, Maron-Katz A, Hendler T, Barrett LF (2016) Functional connectivity dynamics during film viewing reveal common networks for different emotional experiences. Cogn Affect Behav Neurosci 16:709-723. CrossRef Medline

Reitan RM (1958) Validity of the Trail Making Test as an indicator of organic brain damage. Perceptual and Motor Skills 8:271-276.

Rogalski EJ, Gefen T, Shi J, Samimi M, Bigio E, Weintraub S, Geula C, Mesulam MM (2013) Youthful memory capacity in old brains: anatomic and genetic clues from the Northwestern SuperAging Project. J Cogn Neurosci 25:29-36. CrossRef Medline

Schnyer DM, Verfaellie M, Alexander MP, LaFleche G, Nicholls L, Kaszniak AW (2004) A role for right medial prefontal cortex in accurate feelingof-knowing judgements: evidence from patients with lesions to frontal cortex. Neuropsychologia 42:957-966. CrossRef Medline

Seeley WW, Menon V, Schatzberg AF, Keller J, Glover GH, Kenna H, Reiss AL, Greicius MD (2007) Dissociable intrinsic connectivity networks for salience processing and executive control. J Neuroscience 27: 2349-2356

Shenhav A, Botvinick MM, Cohen JD (2013) The expected value of control: an integrative theory of anterior cingulate cortex function. Neuron 79: 217-240. CrossRef Medline

Simons JS, Spiers HJ (2003) Prefrontal and medial temporal lobe interactions in long-term memory. Nat Rev Neurosci 4:637-648. CrossRef Medline

Squire LR (2007) Neuroscience. Rapid consolidation. Science 316:57-58. CrossRef Medline

Squire LR, Stark CE, Clark RE (2004) The medial temporal lobe. Annu Rev Neurosci 27:279-306. CrossRef Medline

Strauss E, Sherman EMS, Spreen O (2006) A compendium of neuropsychological tests: Administration, norms, and commentary (3rd ed) New York, NY: Oxford University Press.

Tombaugh TN (2004) Trail Making Test A and B: normative data stratified by age and education. Arch Clin Neuropsychol 19:203-214. CrossRef Medline

Touroutoglou A, Hollenbeck M, Dickerson BC, Feldman Barrett L (2012) Dissociable large-scale networks anchored in the right anterior insula 
subserve affective experience and attention. Neuroimage 60:19471958. CrossRef Medline

Van Dijk KR, Hedden T, Venkataraman A, Evans KC, Lazar SW, Buckner RL (2010) Intrinsic functional connectivity as a tool for human connectomics: theory, properties, and optimization. J Neurophysiol 103:297-321. CrossRef Medline

Wagner AD (1999) Working memory contributions to human learning and remembering. Neuron 22:19-22. CrossRef Medline

Wang L, Laviolette P, O’Keefe K, Putcha D, Bakkour A, Van Dijk KR, Pihlajamäki M, Dickerson BC, Sperling RA (2010) Intrinsic connectivity between the hippocampus and posteromedial cortex predicts memory performance in cognitively intact older individuals. Neuroimage 51:910917. CrossRef Medline

Weintraub S, Powell DH, Whitla DK (1994) Successful cognitive aging: individual differences among physicians on a computerized test of mental state. J Geriatr Psychiatry 28:15-34.

Wheeler MA, Stuss DT, Tulving E (1997) Toward a theory of episodic memory: the frontal lobes and autonoetic consciousness. Psychol Bull 121: 331-354. CrossRef Medline

Wheeler ME, Buckner RL (2003) Functional dissociation among components of remembering: control, perceived oldness, and content. J Neurosci 23:3869-3880. Medline

Wolk DA, Dickerson BC, Alzheimer's Disease Neuroimaging Initiative (2011) Fractionating verbal episodic memory in Alzheimer's disease. Neuroimage 54:1530-1539. CrossRef Medline

Yaffe K, Fiocco AJ, Lindquist K, Vittinghoff E, Simonsick EM, Newman AB, Satterfield S, Rosano C, Rubin SM, Ayonayon HN, Harris TB (2009) Predictors of maintaining cognitive function in older adults: the Health ABC Study. Neurology 72:2029-2035. CrossRef Medline 